Pacific Journal of Mathematics 


\title{
ON GAMELIN CONSTANTS
}

\author{
MASARU HARA
}

\begin{abstract}
The purpose of this paper is to show that the corona theorem with bounds is valid for any finite bordered Riemann surface. As an application of it we construct an example of Riemann surfaces of infinite genus for which the corona theorem holds. The example can be chosen either from or not from the class of surfaces of Parreau-Widom type.
\end{abstract}

1. Introduction. Let $R$ be a Riemann surface and $H^{\infty}(R)$ be the algebra of bounded analytic functions on $R$. Given a Riemann surface $R$, a natural number $n$ and a positive number $\delta$, we denote by $C_{R}(n, \delta)$ the infimum among constants $C$ having the following property: For any $f_{1}, \ldots, f_{n} \in H^{\infty}(R)$ with $1 \geq \max _{j}\left|f_{j}\right| \geq \delta$ on $R$, there exist $g_{1}, \ldots, g_{n} \in$ $H^{\infty}(R)$ such that $\Sigma_{j} f_{j} g_{j}=1$ on $R$ and $\left|g_{j}\right| \leq C$ on $R(j=1, \ldots, n)$. If there exist no such constants, then we define $C_{R}(n, \delta)=\infty$. We call $C_{K}(n, \delta)$ the Gamelin constant for the triple $(R, n, \delta)$. If $C_{R}(n, \delta)<\infty$ for every $n$ and $\delta>0$, then we say that the Gamelin constant of $R$ is finite.

Gamelin [3] proved that the Gamelin constant of any finitely connected planar domain $R$ is finite in such a way that $C_{R}(n, \delta)$ is dominated by a constant $C_{m}(n, \delta)$ depending only on $n, \delta$ and the number $m$ of boundary components of $R$. The primary purpose of this paper is to prove the following.

THEOREM 1. The Gamelin constant of any Riemann surface which is the interior of any finite bordered Riemann surface is finite.

We raise the question of whether the constants can be chosen to depend only on the genus or rather on the Euler characteristic of the surface.

We denote the maximal ideal space of $H^{\infty}(R)$ by $\Re(R)$. We set $\tau(R)=\{$ the homomorphisms "evaluation at $p$ ": $p \in R\}$. If $H^{\infty}(R)$ separates the points of $R$, we identify $\tau(R)$ with $R$. When $\tau(R)$ is dense in গ $(R)$, we say that the corona theorem holds for $R$. The set $\tau(R)$ is dense in $\mathfrak{T}(R)$ if and only if the following property holds: For each $n$ and $\delta>0$, given $f_{1}, \ldots, f_{n} \in H^{\infty}(R)$ such that $\max \left|f_{j}\right| \geq \delta$ on $R$, there exist $g_{1}, \ldots, g_{n} \in H^{\infty}(R)$ such that $\Sigma_{j} f_{j} g_{j}=1$ on $R$. Therefore if the Gamelin constant of $R$ is finite, then the corona theorem holds for $R$. It is well 
known (e.g. Gamelin [3]) that the corona theorem holds for any finite bordered Riemann surface.

Behrens [1] and Gamelin [3] proved that the corona theorem holds for some infinitely connected planar domains. Cole (cf. Gamelin [4]) gave an example of a Riemann surface for which the corona theorem is not valid. Nakai [6] gave an example of a Riemann surface of Parreau-Widom type for which the corona theorem is invalid. As the second purpose of this paper, we will give an example of a Riemann surface of infinite genus for which the corona theorem holds (Theorem 2). It is obtained from the Behrens example [1]. We will also show that example in Theorem 2 can be chosen from or not from the class of surfaces of Parreau-Widom type (Theorem 3).

2. The proof of Theorem 1. Let $R$ be any finite bordered Riemann surface with genus $g$ and $m$ boundary components. If $g=0$, then Theorem 1 is reduced to the Gamelin theorem. We assume that $g>0$. Let $\gamma_{1}, \ldots, \gamma_{g}$ be simple closed curves on $R$ such that $\gamma_{1}, \ldots, \gamma_{g}$ are mutually disjoint and $R-\cup_{i} \gamma_{i}$ is a plane domain. Let $U_{i}$ be an annulus containing $\gamma_{l}(i=1, \ldots, g)$ such that $\bar{U}_{1}, \ldots, \bar{U}_{g}$ are mutually disjoint. Let $\rho$ be a smooth function on $R$ such that $0 \leq \rho \leq 1$ on $R, \rho=1$ on a neighbourhood of $\cup_{i} \gamma_{i}$ and the support of $\rho$ is contained in $\cup_{i} U_{i}$.

Let $f_{1}, \ldots, f_{n} \in H^{\infty}(R)$ satisfy $1 \geq \max _{j}\left|f_{j}\right| \geq \delta$ on $R$. Since $R-\cup_{i} \gamma_{l}$ is a plane domain of connectivity $2 g+m$, by the Gamelin theorem, there exist $p_{1}, \ldots, p_{n} \in H^{\infty}\left(R-\cup \gamma_{i}\right)$ such that $\sum f_{j} p_{J}=1$ on $R-\cup \gamma_{i}$ and $\max _{j}\left|p_{j}\right| \leq C_{2 g+m}(n, \delta)$. Also since each $U_{i}$ is a plane domain of connectivity 2 , there exist $q_{1}, \ldots, q_{n} \in H^{\infty}\left(\cup U_{i}\right)$ such that $\Sigma f_{j} q_{j}=1$ on $\cup U_{i}$ and $\max _{j}\left|q_{j}\right| \leq C_{2}(n, \delta)$. Set

$$
h_{j}=(1-\rho) p_{j}+\rho q_{j} \quad(j=1, \ldots, n) .
$$

Then $h_{j}$ is smooth on $R$ and $\sum f_{j} g_{j}=1$ on $R$ and $(\partial / \partial \bar{z}) h_{k}=$ $\left(q_{k}-p_{k}\right)(\partial / \partial \bar{z}) \rho$. Set

$$
w_{J k}=\frac{1}{\pi} \iint_{R} C(\zeta, \cdot) h_{J} \frac{\partial}{\partial \bar{z}} h_{k} d \xi d \eta
$$

where $C(\zeta, \cdot)$ is a Cauchy kernel on $R$ which is regular on $\partial R$. Then $(\partial / \partial \bar{z}) w_{j k}=h_{j}(\partial / \partial \bar{z}) h_{k}$. If we set

$$
g_{j}=h_{J}+\sum_{k=1}^{n}\left(w_{j k}-w_{k J}\right) f_{k} \quad(j=1, \ldots, n),
$$


then $g_{j}$ is analytic on $R$ and $\Sigma f_{j} g_{j}=1$. Since

$$
h_{j} \frac{\partial}{\partial \bar{z}} h_{k}-h_{k} \frac{\partial}{\partial \bar{z}} h_{j}=\left(p_{j} q_{k}-p_{k} q_{j}\right) \frac{\partial}{\partial \bar{z}} \rho
$$

if we set $C_{1}=C_{2 g+m}(n, \delta)$ and $C_{2}=C_{2}(n, \delta)$, then

$$
\left|g_{j}\right| \leq C_{1}+C_{2}+2 n C_{1} C_{2} \frac{1}{\pi} \iint\left|C(\zeta, \cdot) \frac{\partial}{\partial \bar{z}} \rho\right| d \xi d \eta
$$

Therefore the Gamelin constant of $R$ is finite.

3. An example. Given a domain $V$ in the complex plane $C$, a sequence $\left\{\Delta_{n}\right\}_{n \geq 1}$ of open disks $\Delta_{n}$ is called a Behrens sequence in $V$ if the following properties hold:

(1) for each $\Delta_{n}$, there exists an open disk $D_{n}$ such that $\bar{\Delta}_{n} \subset D_{n} \subset \bar{D}_{n}$ $\subset V$ and $\Delta_{n}$ and $D_{n}$ have the common center $\alpha_{n}$;

(2) $d\left(\alpha_{n}, \partial V\right) \rightarrow 0$ as $n \rightarrow \infty$, where $d$ is the Riemann spehre metric;

(3) the disks $\bar{D}_{n}$ in $\left\{D_{n}\right\}$ are mutually disjoint;

(4) $\Sigma\left(\operatorname{rad} \Delta_{n}\right) /\left(\operatorname{rad} D_{n}\right)<\infty$, where $\operatorname{rad} \Delta$ is the radius of $\Delta$;

(5) $\left(\operatorname{rad} D_{n}\right) / d(\alpha, \partial V) \rightarrow 0$ as $n \rightarrow \infty$.

Behrens [1] proved that if the corona theorem holds for $V$ and $\left\{\Delta_{n}\right\}$ is a Behrens sequence of disks in $V$, then the corona theorem holds for the domain $U=V-\cup_{n} \bar{\Delta}_{n}$, which is called the region $V$ with a Behrens sequence $\left\{\Delta_{n}\right\}$ in $V$ removed.

let $\left\{\Delta_{n}\right\}$ be a Behrens sequence of disks in $V$. We denote by $\tilde{\Delta}_{n}$ a copy of $\Delta_{n}$ for each $n$. We introduce into each $\Delta_{n}$ a finite number $(\geq 2)$ of mutually disjoint slits. Each slit is considered to have two banks: an $N$-bank and an $S$-bank. By joining every $S$ - (resp. $N$-) bank of slits on $\Delta_{n}$ to an $N$ - (resp. $S$-) bank of the corresponding slits on $\tilde{\Delta}_{n}$, we can construct a two sheeted covering Riemann surface of $\Delta_{n}$ which will be denoted by $\Delta_{n}+\tilde{\Delta}_{n}$. We assume that any two members in $\left\{\Delta_{n}+\tilde{\Delta}_{n}\right\}$ are mutually conformally equivalent. By welding the two sheeted disk $\Delta_{n}+\tilde{\Delta}_{n}$ to the Behrens domain $U=V-\cup_{n} \Delta_{n}$ along the boundary $\partial \Delta_{n}$ of $\Delta_{n}+\tilde{\Delta}_{n}$ and the boundary of $U$ where $\bar{\Delta}_{n}$ is removed, we obtain a Riemann surface $R=V+\cup_{n} \tilde{\Delta}_{n}=U+\cup_{n}\left(\Delta_{n}+\tilde{\Delta}_{n}\right)$, which is called the Riemann surface $V$ with a Behrens sequence $\left\{\Delta_{n}\right\}$ in $V$ attached.

We are ready to state the following.

THEOREM 2. If the corona theorem holds for a domain $V$ in the complex plane $C$, then the corona theorem holds for the Riemann surface $V$ with a Behrens sequence $\left\{\Delta_{n}\right\}$ in $V$ attached. 
Let $\hat{C}=C \cup\{\infty\}$. We consider projections $P_{n} f$ of each function $f$ in $H^{\infty}(R)$ to $H^{\infty}\left(\hat{C}+\tilde{\Delta}_{n}\right)$ by the following: First let

$$
P_{n} f(z)=\frac{-1}{2 \pi i} \int_{\partial \Delta_{n}} \frac{f(\zeta)}{\zeta-z} d \zeta
$$

for $z$ in $\hat{C}-\bar{\Delta}_{n}$. Observe

$$
P_{n} f(z)=f(z)-\frac{1}{2 \pi i} \int_{\partial D_{n}} \frac{f(\zeta)}{\zeta-z} d \zeta
$$

for $z$ in $D_{n}-\bar{\Delta}_{n}$. Since the right hand side of the above may be considered as a holomorphic function on $D_{n}+\tilde{\Delta}_{n}$, we can consider that $P_{n} f \in H^{\infty}\left(\hat{C}+\tilde{\Delta}_{n}\right)$. By Lemma 2.1 of Behrens [1], $\Sigma P_{n} f$ converges normally to a bounded analytic function, and therefore $f-\sum_{n=1}^{\infty} P_{n} f \in$ $H^{\infty}(V)$. We have thus established the following decomposition:

$$
H^{\infty}(R)=\sum_{n} H^{\infty}\left(\hat{C}+\tilde{\Delta}_{n}\right)+H^{\infty}(V) .
$$

Moreover, by Theorem 1, the Gamelin constant of $\Delta_{n}+\tilde{\Delta}_{n}$ is finite. Since any two members in $\left\{\Delta_{n}+\tilde{\Delta}_{n}\right\}$ are mutually conformally equivalent, the Gamelin constants of $\Delta_{n}+\tilde{\Delta}_{n}$ are all the same. The Behrens result [1] corresponding to Theorem 2 was proved based upon a decomposition corresponding to the above decomposition of $H^{\infty}(R)$ and the fact that Gamelin constants of removing disks are all the same finite constant. Since we have all the corresponding necessary machinery, we can repeat almost the same argument used by Behrens [1] to complete the proof of Theorem 2. We omit the details.

Next we will prove the following

THEOREM 3. The Riemann surface $D+\cup_{n} \tilde{\Delta}_{n}$ can be made either of Parreau-Widom type or not by the choice of the Behrens sequence $\left\{\Delta_{n}\right\}$ in the unit disk $D$.

Set $\log \left(1 / r_{m}\right)=2^{-m}(m=1,2, \ldots)$. We give a Behrens sequence $\left\{\Delta_{n}\right\}$ in $D$ as follows. The first $p_{1}$ number of $D_{n}$ 's have centers $\alpha_{n}$ on $\left\{|z|=r_{1}\right\}$, the next $p_{2} D_{n}$ 's have centers $\alpha_{n}$ on $\left\{|z|=r_{3}\right\}$, etc., and all $D_{n}$ 's are disjoint from the circles $\left\{|z|=r_{2 m}\right\}, 1 \leq m<\infty$. Let $g$ be the Green's function of $D+\tilde{\Delta}_{n}$ with its pole at $z=0$. Let $\pi$ be the projection of $\Delta_{n}+\tilde{\Delta}_{n}$ onto $\left\{\left|z-\alpha_{n}\right|<\operatorname{rad} \Delta_{n}\right\}$. We denote by $u(z)$ the harmonic function on $\Delta_{n}+\tilde{\Delta}_{n}$ which is equal to $\log (1 /|z|)$ on $\partial \Delta_{n}$ and 0 on $\partial \tilde{\Delta}_{n}$. Then on the disk $\left\{\left|z-\alpha_{n}\right|<\operatorname{rad} \Delta_{n}\right\}, u\left(z_{1}\right)+u\left(z_{2}\right)=\log (1 /|z|)$ where 
$\pi^{-1}(z)=\left\{z_{1}, z_{2}\right\}$. The function which is equal to $\log (1 /|z|)$ on $D-\bar{\Delta}_{n}$ and $u(z)$ on $\Delta_{n}+\tilde{\Delta}_{n}$ is superharmonic on $D+\tilde{\Delta}_{n}$. Hence $\log (1 /|z|) \geq g$ on $D-\bar{\Delta}_{n}$. If $\operatorname{rad} \Delta_{n}$ is sufficiently small, then we have $\log (1 /|z|) \geq g$ $\geq \frac{1}{4} \log (1 /|z|)$ on $D-\bar{D}_{n}$. We denote by $G$ the Green's function of $D+\cup_{n} \tilde{\Delta}_{n}$ with its pole at $z=0$. By the above argument, if each term of $\left\{\operatorname{rad} \Delta_{n}\right\}$ is sufficiently small, then we have $\log (1 /|z|)>G>\frac{1}{4} \log (1 /|z|)$ on $D-\cup_{n \geq 1} \bar{D}_{n}$. The open set $\left(D+\cup_{n} \tilde{\Delta}_{n}\right)-\left\{|z|=r_{2 m}\right\}$ consists of two components, one of which containing the center of $D$ will be denoted by $R_{m}$. Then $\left\{|z|<r_{2 m-2}\right\}-\cup_{n \geq 1} \bar{D}_{n} \subset\left\{G>4^{-m}\right\} \subset R_{m}$. By the maximum principle, the complement of $\left\{G>4^{-m}\right\}$ does not contain any compact component. Therefore if a cycle in $\left\{G>4^{-m}\right\}$ is homologous to zero in $R_{m}$, then it is homologous to zero in $\left\{G>4^{-m}\right\}$. Hence we have

$$
p_{1}+\cdots+p_{m-1} \leq B\left(0,4^{-m}\right) \leq b\left(p_{1}+\cdots+p_{m}\right),
$$

where $b($ resp. $B(0, \alpha))$ is the first Betti number of $\Delta_{n}+\tilde{\Delta}_{n}($ resp. $\{G>\alpha\})$. Since any two members in $\left\{\Delta_{n}+\tilde{\Delta}_{n}\right\}$ are mutually conformally equivalent, $b$ does not depend on $n$. Hence

$$
\int_{0}^{\infty} B(0, \alpha) d \alpha<\infty \quad \text { if and only if } \sum_{m \geq 1} 4^{-m}\left(p_{1}+\cdots+p_{m}\right)<\infty .
$$

By the Widom theorem (cf. Widom [7], [8]), $D+\cup \tilde{\Delta}_{n}$ is of ParreauWidom type if and only if $\Sigma_{n \geq 1} 4^{-m}\left(p_{1}+\cdots+p_{m}\right)<\infty$.

\section{REFERENCES}

[1] M. Behrens; The maximal ideal space of algebras of bounded analytic functions on infinitely connected domains, Trans. Amer. Math. Soc., 161 (1971), 359-380.

[2] L. Carleson, Interpllation by bounded analytic functions and the corona problem, Ann. of Math., 76 (1962), 547-559.

[3] T. Gamelin, Localization of the corona problem, Pacific J. Math., 34 (1970), 73-81.

[4] Uniform algebras and Jensen measures, London Math. Soc. Lecture Note Series 32, Cambridge Univ. Press, 1978.

[5] __, Wolff's proof of the corona theorem, Israel J. Math., 37 (1980), 113-119.

[6] M. Nakai, Corona problem for Riemann surfaces of Parreau-Widom type, Pacific J. Math., 103 (1982), 103-109.

[7] H. Widom, The maximum principle for multiple-valued analytic functions, Acta Math., 126 (1971), 63-82.

[8] $\longrightarrow H_{p}$ sections of vector bundles over Riemann surfaces, Ann. of Math., 94 (1971), 305-324.

Received August 26, 1981 and in revised form November 19, 1982. 



\section{PACIFIC JOURNAL OF MATHEMATICS \\ EDITORS}

DONALD BABBITT (Managing Editor)

University of California

Los Angeles, CA 90024

Hugo RossI

University of Utah

Salt Lake City, UT 84112

C. C. MOOre and ARThur OGus

University of California

Berkeley, CA 94720
J. Dugundi

Department of Mathematics

University of Southern California

Los Angeles, CA 90089-1113

R. FINN and H. SAMELSON

Stanford University

Stanford, CA 94305

ASSOCIATE EDITORS

$\begin{array}{lllll}\text { R. Arens } & \text { E. F. Beckenbach } & \text { B. H. Neumann } & \text { F. Wolf } & \text { K. Yoshida }\end{array}$ $(1906-1982)$

\section{SUPPORTING INSTITUTIONS}

UNIVERSITY OF ARIZONA

UNIVERSITY OF BRITISH COLUMBIA

CALIFORNIA INSTITUTE OF TECHNOLOGY

UNIVERSITY OF CALIFORNIA

MONTANA STATE UNIVERSITY

UNIVERSITY OF NEVADA, RENO

NEW MEXICO STATE UNIVERSITY

OREGON STATE UNIVERSITY
UNIVERSITY OF OREGON

UNIVERSITY OF SOUTHERN CALIFORNIA

STANFORD UNIVERSITY

UNIVERSITY OF HAWAII

UNIVERSITY OF TOKYO

UNIVERSITY OF UTAH

WASHINGTON STATE UNIVERSITY

UNIVERSITY OF WASHINGTON 


\section{Pacific Journal of Mathematics}

\section{Vol. 110, No. $1 \quad$ September, 1984}

Wojciech Abramczuk, A class of surjective convolution operators $\ldots \ldots \ldots \ldots 1$

K. Adachi, Extending bounded holomorphic functions from certain

subvarieties of a weakly pseudoconvex domain $\ldots \ldots \ldots \ldots \ldots \ldots$

Malvina Florica Baica, An algorithm in a complex field and its application

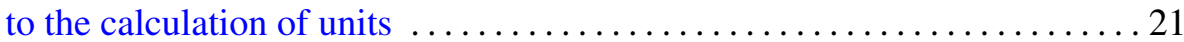

Giuliana Bianchi and Robert Cori, Colorings of hypermaps and a

conjecture of Brenner and Lyndon $\ldots \ldots \ldots \ldots \ldots \ldots \ldots \ldots \ldots \ldots \ldots \ldots$

Ronald James Evans, Determinations of Jacobsthal sums . . .......... 49

Leslie Foged, Characterizations of $\aleph$-spaces .................... 59

Nassif A. Ghoussoub and Paulette Saab, Weak compactness in spaces of

Bochner integrable functions and the Radon-Nikodým property . . . . . . 65

J. Gómez Gil, On local convexity of bounded weak topologies on Banach

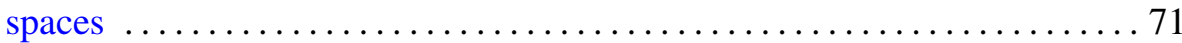

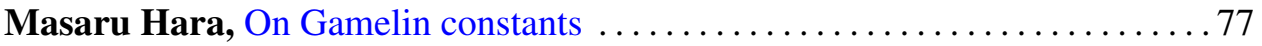

Wilfried Hauenschild, Eberhard Kaniuth and Ajay Kumar, Harmonic

analysis on central hypergroups and induced representations $\ldots \ldots \ldots 83$

Eugenio Hernandez, An interpolation theorem for analytic families of

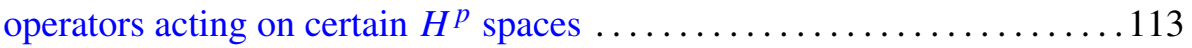

Thomas Alan Keagy, On "Tauberian theorems via block-dominated

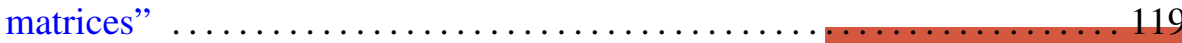

Thomas Landes, Permanence properties of normal structure $\ldots \ldots \ldots \ldots \ldots 125$

Daniel Henry Luecking, Closed ranged restriction operators on weighted

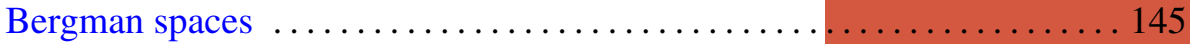

Albert Thomas Lundell, The $p$-equivalence of $\mathrm{SO}(2 n+1)$ and $\mathrm{Sp}(n) \ldots \ldots 161$

Mark D. Meyerson, Remarks on Fenn's "the table theorem" and Zaks' "the chair theorem" ..................................... 167

Marvin Victor Mielke, Homotopically trivial toposes . . . . . . . . . . 171

Gerard J. Murphy, Hyperinvariant subspaces and the topology on Lat A . . 183

Subhashis Nag, On the holomorphy of maps from a complex to a real manifold

Edgar Milan Palmer and Robert William Robinson, Enumeration of self-dual configurations ................................. 203

John J. Walsh and David Clifford Wilson, Continuous decompositions

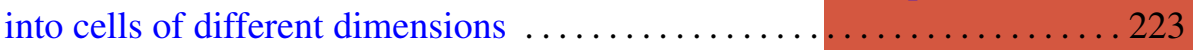

Walter John Whiteley, Infinitesimal motions of a bipartite framework .....233 